\title{
Free recall measures of short-term store: Are rehearsal and order of recall data necessary?
}

\author{
DELBERT A. BRODIE
}

\author{
University of Western Ontario, London, Ontario, Canada N6A 5C2
}

\begin{abstract}
Several investigators have claimed that immediate free recall is a composite of output from two different storage systems-a short-term store (STS) and a long-term store (LTS). Free recall data and measures of STS were evaluated by having subjects report their rehearsals while lists of words were presented at a rate of one word every $1.25,2.50$, or $5.00 \mathrm{sec}$. The results support the conclusions that (a) arranging recall as a function of where an item was presented, rather than where it was rehearsed, is inappropriate to discussions of STS and LTS, and (b) computing measures of STS without both rehearsal and order of recall data yields a biased estimate of STS.
\end{abstract}

Several investigators (Atkinson \& Shiffrin, 1968; Craik, 1971; Glanzer, 1972; Watkins, 1974) have hypothesized that immediate free recall is a composite of output from two storage systems. The probability that an item is output from one of these stores has been assumed to decline rapidly as a function of either time (Glanzer, 1972, p. 145; Glanzer \& Cunitz, 1966; Reitman, 1974) or number of different items (Glanzer, Gianutsos, \& Dubin, 1969; Waugh \& Norman, 1965) intervening between last rehearsal of a given item and its recall. As a result of rapid decay, this hypothetical store has been called a "short-term store" (STS). In contrast to STS, the probability that an item is in the other store has been assumed to remain relatively constant as a function of these manipulations (Craik, 1971; Glanzer \& Cunitz, 1966). Hence, the latter hypothetical store has been called a "long-term store" (LTS).

Some evidence which has been alleged to support the existence of separate STS and LTS in free recall tasks has consisted of the finding that certain manipulations affect the beginning and middle of the free recall curve differently from the end. . For instance, increases in presentation time augment immediate free recall of preterminal items, but not terminal items (Glanzer \& Cunitz, 1966; Murdock, 1962). These findings have been alleged to support the existence of separate stores because both time and number of items intervening between item presentation and list termination decrease with position. Hence, it seemed reasonable to assume that both time and number of items intervening between last rehearsal of an item and its recall would also

This research was supported by a National Research Council of Canada postgraduate scholarship to the author, who is now at the University of Toronto, Toronto, Ontario, Canada M5S 1A1. decrease with position. If this occurred, and if the probability of STS-P(STS)-decays as a function of either increases in intervening time or number of items, then P(STS) should increase with position. Hence, differential effects of manipulations on the free recall curve appear to be explained by assuming that the manipulations affect $P(S T S)$ differently than P(LTS).

Ideally, if hypothetical STS and LTS exist, then, rather than merely determining whether various manipulations have different effects on immediate free recall of preterminal and terminal items, one should obtain estimates of $\mathrm{P}(\mathrm{STS})$ and P(LTS). Several procedures have been proposed to do this. Some of these procedures assume that all items in storage are recalled. If this is correct, then the probability that an item will be recalled immediately following list presentation equals the probability that an item is in STS and not LTS, in LTS and not STS, or in both STS and LTS,

$$
\begin{aligned}
& P(I)= \\
& P(S T S \cap \overline{L T S})+P(L T S \cap \overline{S T S})+P(S T S \cap L T S) .
\end{aligned}
$$

One can derive an indirect measure of STS from Equation 1 by first estimating P(LTS) and then solving for $\mathrm{P}(\mathrm{STS})$. Three indirect measures of STS have been reported. The assumptions underlying one of these measures (Baddeley, Scott, Drynan, \& Smith, 1969; Raymond, 1969) are that an item cannot be in both STS and LTS concurrently, and delaying recall by engaging the subject in a "rehearsal prevention" task for 15 to $60 \mathrm{sec}$ reduces $\overline{\mathrm{P}}(\overline{\mathrm{S} T S} \cap \overline{\mathrm{LTS}})$ to zero without altering P(LTS $\cap \overline{\text { STS }})$. Consequently, 
$P(S T S)$ equals $P(I)$ minus the probability of delayed recall- $P(D)$.

The two remaining indirect measures of STS differ from the first measure by assuming that an item can be in both STS and LTS concurrently. Hence, even though $\mathrm{P}(\mathrm{STS})$ is assumed to rapidly decline after last rehearsal of an item, P(STS) is identified with both stable and unstable components of the subject's recall since items output from STS immediately following list presentation may be later output from LTS.

To deduce the two remaining indirect measures, it is necessary to rewrite Equation 1 as

$$
\mathrm{P}(\mathrm{I})=\mathrm{P}(\mathrm{STS})+\mathrm{P}(\mathrm{LTS})-\mathrm{P}(\mathrm{STS} \cap \mathrm{LTS}) .
$$

where $\mathrm{P}(\mathrm{STS})$ and $\mathrm{P}(\mathrm{LTS})$ each represent the probability that an item is in only one memory store plus the probability that the item is in both stores concurrently. The P(STS $\cap$ LTS) equals P(STS) times P(LTS) if the probability that an item is in STS is independent of the probability that it is in LTS. Hence, by assuming independence, Equation 2 can be rewritten as

$$
\mathrm{P}(\mathrm{STS})=\frac{\mathrm{P}(\mathrm{I})-\mathrm{P}(\mathrm{LTS})}{1-\mathrm{P}(\mathrm{LTS})}
$$

Two procedures have been used to solve Equation 3 by specifying different methods for computing $P($ LTS). The assumptions underlying one of these procedures (Glanzer \& Schwartz, 1971; Raymond, 1969) are that engaging the subject in a "rehearsal prevention" task for up to $60 \mathrm{sec}$ reduces P(STS) to

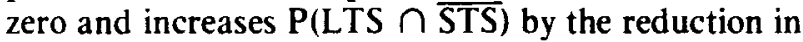
$\mathrm{P}(\mathrm{STS} \cap \mathrm{LTS})$. Hence, $\mathrm{P}(\mathrm{LTS})$ equals $\mathrm{P}(\mathrm{D})$, and P(STS) equals $[P(I)-P(D)] /[1-P(D)]$. Occasionally, this formula for STS has been expressed as (I - D) $/(\mathbf{N}-\mathbf{D})$, where $\mathbf{N}$ is the denominator of $\mathrm{P}(\mathrm{I})$ and $P(D)$, and $I$ and $D$ are the numerators (Baddeley, 1970; Levy \& Baddeley, 1971).

The assumptions underlying the other procedure for solving Equation 3 (Waugh \& Norman, 1965) are that immediately following presentation of a long list, only terminal items are in STS, and any change in $\mathrm{P}(\mathrm{STS} \cap \mathrm{LTS})$ across middle and terminal items is accompanied by an equal change in magnitude, but opposite direction, in P(LTS $\cap$ STS). Hence, P(LTS) remains constant across middle and terminal items, and $P$ (LTS) for terminal items is equal to the average probability of recalling middle items-P(M)immediately after list presentation. Consequently, $P(S T S)$ equals $[P(I)-P(M)] /[1$ - $P(M)]$.

The above indirect measures of STS are currently the most frequently reported measures of STS. This is surprising because some "proponents of these measures (Glanzer, 1972; Raymond, 1969) state that they want to separate the probability of output from STS and LTS. Indirect measures, however, supposedly indicate the probability that an item is in STS, and not necessarily the probability that an item is output from STS.

If items can be in both stores concurrently. then the probability that an item is output from each store would equal the probability that an item is in each store if and only if (a) items can be output from both stores concurrently, and (b) the probability that an item is output from one store is independent of its being output from the other store. It has been maintained (Craik \& Levy, 1970) that in some situations items in both stores may be output from LTS rather than STS. However, if items in STS are readily accessible whereas items in LTS are accessible only after the subject searches for them (Atkinson \& Shiffrin. 1971; Craik, 1968), then in free recall tasks items in both stores should be output solely from STS. If this occurs, then the probability of outputting an item from STS equals the probability that the item is in STS. while the probability of outputting the item from LTS immediately following list presentation equals $P(I)$ minus $P(S T S)$.

If items in STS are output prior to items in LTS, then, rather than computing indirect measures of STS, one could compute a direct measure by calculating the probability that an item is one of the first items recalled. Alternatively, one could try to ensure that the first items recalled are output from STS by computing a last-presented/first-recalled measure where P(STS) equals the probability that items presented immediately prior to list termination are recalled prior to items which were presented earlier in the list. Occasionally, subjects have been instructed to begin recall with the last-presented items (Anderson \& Craik, 1974; Craik \& Levy, 1970). Unfortunately, however, neither a first-recall nor a last-presented/first-recalled measure has been reported in previous free recall experiments.

Previously reported direct measures that have been used to compute P(STS) for individual items have been limited by investigators assuming that only the last items presented are in STS, and that some items solely in LTS may be recalled prior to items in STS. As a result of making these assumptions, some investigators (Glanzer \& Cunitz, 1966; Tulving \& Patterson. 1968) have used a recency measure where $\mathrm{P}(\mathrm{STS})$ equals the probability of recalling terminal items immediately following list presentation regardless of their order of recall. On the other hand, other investigators (Craik. 1971; Craik \& Birtwistle. 1971; Tulving \& Colotla, 1970) have used an intervening item measure where $P(S T S)$ equals the probability that terminal items are recalled with less than a specified number of items intervening between presentation and recall of each terminal item. Intervening item measures of STS can be subdivided into an intervening different-item measure where each intervening item is different from all other intervening 
items, and an intervening same-item measure where the same item is counted as two intervening items if it is both presented after and recalled before a given item.

The last direct measures of STS differ from all other measures in that they have not been used to calculate P(STS) for individual items. Instead, they have been used to indicate the average P(STS) across all positions or the total number of items in STS. Specifically, an intercept measure (Craik, 1968; Murdock, 1960, 1967) states that the number of items in STS equals the intercept of the least squares solution to the best fitting straight line through points representing the number of items recalled as a function of total presentation time. On the other hand, an intersection measure (Craik, 1968) states that the number of items in STS equals the intersection of the line representing perfect recall as a function of list length with the line representing actual recall.

The purpose of the present experiment was to determine which measures of STS are the best measures of theoretical STS. This was accomplished by examining the effects of three variables.

One of the variables examined was the effect of presentation time on recall, measures of STS, and both time and number of different items intervening between last rehearsal of an item and list termination. The effect of presentation time on intervening time and different items was examined because memory dichotomy theorists have assumed that the probability that an item is in STS varies as a function of these factors. Hence, data on the effect of presentation time on intervening time and different items will indicate how presentation time should affect measures of STS. Several measures which have been used to record changes in STS were computed to determine which measures exhibit the expected effect of presentation time on theoretical STS.

One problem with comparing several measures is that prior to computing $\mathbf{P}(\mathrm{STS})$, investigators have imposed different arbitrary limits on different measures. In order to eliminate the possibility that differences among measures could arise from different arbitrary limitations on the maximum number of items output from STS, all investigatorbounded measures (except first recall) were defined such that the maximum number of items output from STS was six. Thus, for example, an item was counted as being output from STS using an intervening item measure if and only if five or less items intervened between presentation and recall of that item. Similarly, an item was counted as being output from STS using a last-presented/first-recalled measure if and only if the item was one of the last six items presented and it was recalled immediately following list presentation prior to items which were not one of the last six presented. Six was chosen as the maximum number of items output from STS using most investigator-bounded measures because the maximum number of items the subject can recall with complete accuracy after one presentation is approximately six (Atkinson \& Shiffrin, 1968, p. 112).

The only investigator-bounded measure assigned a limit different from six was first recall. When the firstrecall measure was limited to six, it was the only investigator-bounded measure that failed to restrict STS to the last items subjects rehearsed. In order to determine whether this failure was due to placing a high limit on the maximum number of items output from STS, the limit placed on the first-recall measure was reduced to three items.

The second variable examined in the present experiment was the effect of arranging data as a function of both an item's nominal position and its functional position during list presentation. Nominal position refers to the number of different list items which were presented prior to presentation of a given item. Similarly, functional position refers to the number of different list items which received their last rehearsal prior to last rehearsal of a given item. Previously, data which has been alleged to support the existence of separate STS and LTS have been arranged only as a function of an item's nominal position. However, as noted above, memory dichotomy theorists have assumed that the probability that an item is in STS varies as a function of either time or number of different items intervening between last rehearsal and recall of that item. Hence, the conventional arrangement of data is appropriate to discussions of STS only if similar results are obtained when data are arranged as a function of an item's functional position.

Finally, the third variable examined in the present experiment was the effect of computing P(STS) with presentation and rehearsal data. Previously, P(STS) has been computed only with presentation data. Thus, for example, a recency measure has been computed for the last six items presented rather than for the last six items rehearsed. Similarly, a $P(I)-P(D)$ measure has been computed by estimating $P(D)$ for a given item from delayed recall of another item which was presented in the same nominal position as the given item rather than from delayed recall of an item which received its last rehearsal in the same functional position as the given item. Whether previous computations of STS are valid depends on whether one accepts the claim made by memory dichotomy theorists (Atkinson \& Shiffrin, 1968; Waugh \& Norman, 1965) that each rehearsal generates a STS trace. If this is correct, then using presentation data to compute at least some measures of STS such as recency, intervening item, and 
$[\mathrm{P}(\mathrm{I})-\mathrm{P}(\mathrm{M})] /[1-\mathrm{P}(\mathrm{M})]$ is valid only if presentation data yield the same results as those obtained with rehearsal data.

\section{METHOD}

\section{Subjects}

Thirty-six introductory psychology students at the University of Western Ontario participated to fulfill a course requirement.

\section{Materials and Apparatus}

One-syllable nouns with a running frequency between 50 and 300 occurrences per million (both before and after the forms of each noun were combined using the procedure described by Thorndike and Lorge, 1944, p. ix) were selected randomly from Kučera and Francis (1967). The nouns were assigned randomly without replacement to 3 practice lists and 12 experimental lists. Each list contained six beginning, six middle, and six terminal items. An item was classified as being a beginning, middle, or terminal item based on where it was presented or where it was rehearsed. For example, a terminal item was either one of the last six items presented or one of the last six items rehearsed.

The lists were presented on a memory drum at a rate of one item every $1.25,2.50$, or $5.00 \mathrm{sec}$. A tape recorder was used to record the sound of the memory drum advancing and subjects' vocalizations.

\section{Procedure}

Individual subjects were shown each list once followed by a 1 -min written free recall test. For each subject, items in each practice list were presented for a different presentation time, and on at least one practice list, time between list termination and the signal to begin recall (that is, the list retention interval) was $0 \mathrm{sec}$, whereas on another practice list the list retention interval was $15 \mathrm{sec}$. On the experimental lists, presentation time and list retention interval were counterbalanced across trials within subjects and across both trials and lists between subjects.
At the start of the experiment, each subject was instructed to continually report during list presentation which list items he was thinking about (Corballis, 1969; Rundus \& Atkinson, 1970). No restrictions were placed on either the choice of list items to be rehearsed or how the subject tried to encode them. At the start of each trial, the subject was told how fast the items would be presented. Following list presentation, each subject was instructed to either recall immediately or count backwards by threes for $15 \mathrm{sec}$ and then recall.

\section{RESULTS}

As specified by Myers (1972, pp. 176-179), F statistics were evaluated using ultraconservative degrees of freedom for $\mathrm{F}$ statistics which are clearly significant, and ultraliberal degrees of freedom for $F$ statistics which are clearly nonsignificant. Whenever these degrees of freedom failed to indicate unambiguously whether an $\mathrm{F}$ statistic was significant or not significant, the degrees of freedom were adjusted for the observed heterogeneity of variances and covariances. Only F statistics which were not qualified by any higher order interactions are reported below.

\section{Recall, Time, and Different Items}

Recall, time, and different item data were analyzed by 2 by 2 by 2 by 3 by 9 analyses of variance-each with arrangement (nominal or functional), list retention interval, immediate $(0 \mathrm{sec})$ or delayed $(15 \mathrm{sec}$ ), trials (first or second), presentation time
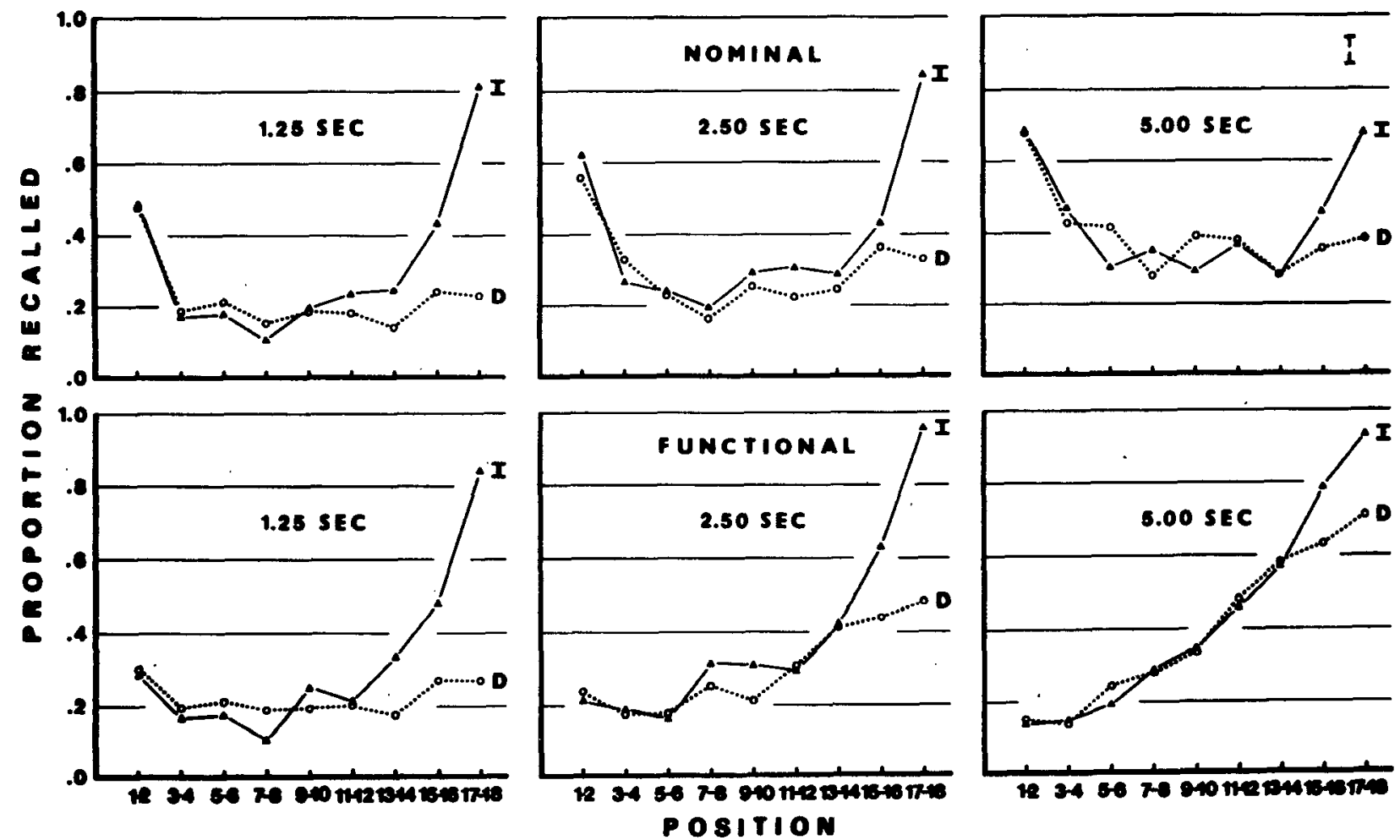

Figure 1. Proportion of items recalled as a function of arrangament (nominator functional), presentation time (1.25, 2.50, or 5.00 ec), and position when recall was either immediate (I) or delayed (D). Since the variances were homogeneous, \pm 1 standard error of the mean was averaged across all points. The broken vertical line represents the avertige standard exror of the mean. 


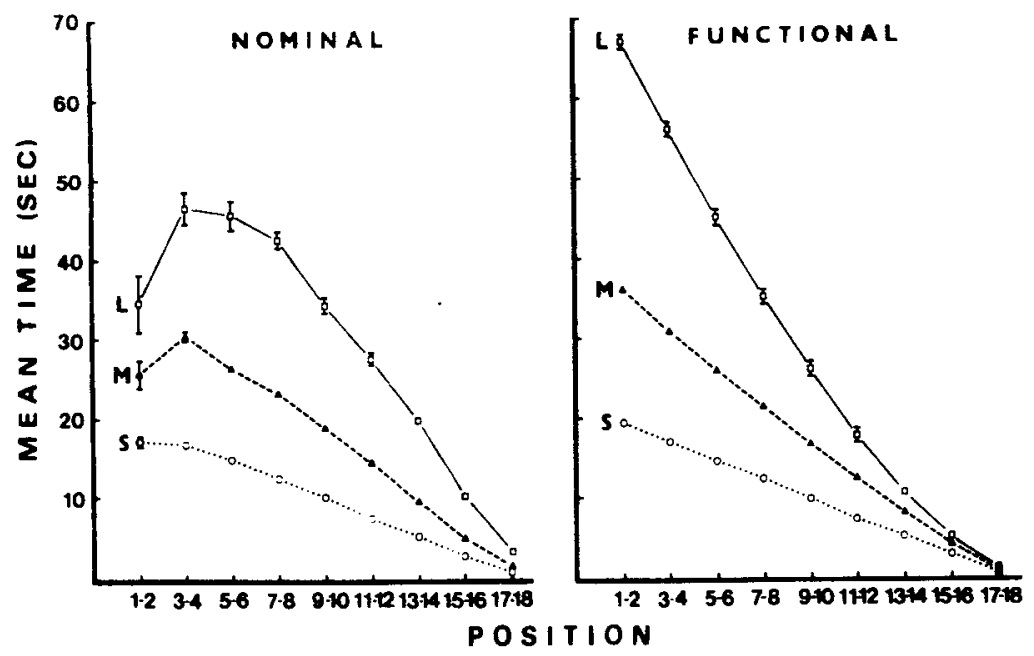

Figure 2. Mean time between last rehearsal of an item and list termination as a function of presentation time $(L=$ long $=\mathbf{5 . 0 0} \mathrm{sec}, M=$ moderate $=2.50 \mathrm{sec}$, or $\mathrm{S}=$ short $=$ $1.25 \mathrm{sec}$ ), arrangement (nominal or functional), and position. The vertical lines represent \pm 1 standard error of the mean. The absence of vertical lines indicates that the standard error lies within the area encompassed by the data point.

(1.25, 2.50. or $5.00 \mathrm{sec}$ ). and position (1 to 18 with two adjacent positions averaged) as within-subject factors. Analysis of recall data indicated that the List Retention Interval by Position. $F(1.35)=29.07$. $p<.01$. List Retention Interval by Presentation Time. $F(2.47)=4.50 . p<.05$, and Arrangement by Presentation Time by Position. $F(1.35)=15.62$. $\mathrm{p}<.01$. interactions were significant. Inspection of Figure 1 indicates that the List Retention Interval by Position interaction resulted from recall of items in the last few positions declining with a small increase in the list retention interval. whereas recall of items in preterminal positions remained constant. Inspection of Figure 1 also indicates that the List Retention Interval by Presentation Time interaction was attributable to an increase in list retention interval producing larger reductions in recall when items were presented either every $1.25 \mathrm{sec}$ or every $2.50 \mathrm{sec}$ than when items were presented every $5.00 \mathrm{sec}$. Finally, inspection of Figure 1 indicates that the Arrangement by Presentation Time by Position interaction resulted from increases in presentation time increasing recall of items in preterminal positions more than items in terminal positions with a nominal arrangement. whereas the reverse occurred with a functional arrangement.

Like analysis of recall, analyses of time, $F(1.35)=$ 71.66. and number of different items rehearsed, $F(1.35)=35.24$, between last rehearsal of an item and list termination indicated that the Arrangement by Presentation Time by Position interactions were significant $(p<.01)$. These interactions are illustrated in Figures 2 and 3. Inspection of Figure 2 indicates that placing items in their functional rather than nominal positions increased time between last rehearsal and list termination for items in beginning positions while decreasing time for items in terminal positions when itens were presented for long rather than short presentation times. Inspection of Figure 3 indicates that increases in presentation tinue decreased the number of different items intervening between last rehearsal of items in beginning nominal positions and list termination while increasing the number of intervening items for items in middle and terminal nominal positions. By definition, when items were in their functional rather than nominal positions, the number of different items intervening between last rehearsal of an item and list termination decreased linearly from 16.5 for the first two items to 0.5 for the last two items.

\section{Measures of STS}

Examination of published experiments indicates that everyone averages some recall scores prior to computing $[\mathrm{P}(\mathrm{I})-\mathrm{P}(\mathrm{D})] /[1 \quad \mathrm{P}(\mathrm{D})]$ and $[P(I)-P(M)] /[1-P(M)]$ measures of STS. The number of recall scores averaged, however, varies from

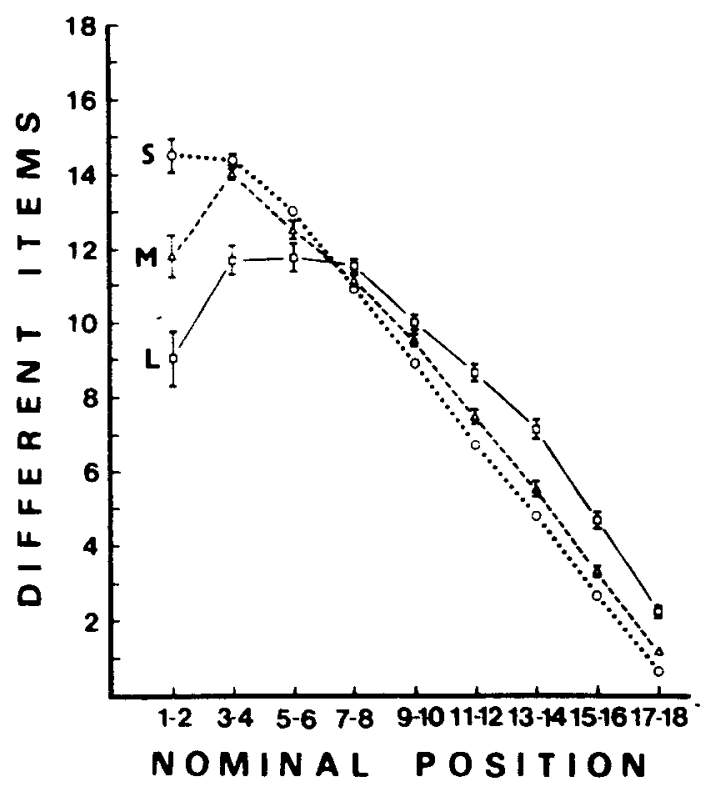

Figure 3. Mean number of different list items rehearsed between last rehearsal of an item and list termination as a function of presentation time $(S=$ short $=1.25 \mathrm{sec}, M=$ moderate $=$ $2.50 \mathrm{sec}$, or $\mathrm{L}=$ long $=\mathbf{5 . 0 0} \mathrm{sec}$ ) and nominal position. The vertical lines represent \pm 1 standard error of the mean. The absence of vertical lines indicates that the standard error lies within the area encompassed by the data point. 


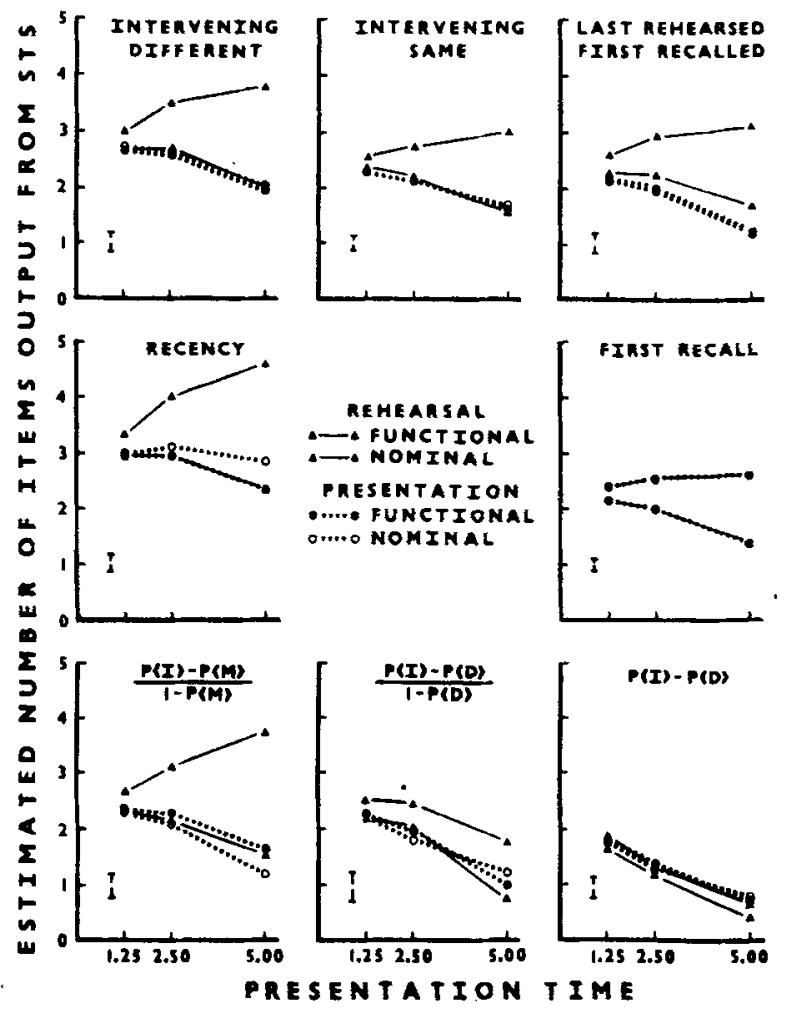

Figure 4. Estimated number of the last six items output from STS as a function of data (rehearsal or presentation), arrangemeat (functional or nommal), measure (intervening different-item, intervening same-item, last-rehearsed/firat-recalled, recency, first-recall, $[\mathbf{P}(\mathbf{I})-\mathbf{P}(\mathbf{M})] /[1$ - $\mathbf{P}(\mathbf{M})],[\mathbf{P}(\mathbf{I})-\mathbf{P}(\mathbf{D})] /[1-\mathbf{P}(\mathrm{D})]$, or $\mathbf{P}(I)-\mathbf{P}(D))$, and presentation time. Since the variances were homogeneous across all variables except measure, \pm 1 standard error of the mean was averaged across means for each menuure. The broken vertical lines represent the avernge standard error of the mean for each measure.

experiment to experiment. For example, prior to computing $[\mathrm{P}(\mathrm{I})-\mathrm{P}(\mathrm{D})] /[1$ - $\mathrm{P}(\mathrm{D})]$, Raymond (1969) averaged recall of 4 items, Silverstein and Glanzer (1971) averaged recall of 9 items, and Glanzer and Schwartz (1971) averaged recall of 16 items. In the present experiment, the average number of items in STS using a $[P(I)-P(D)] /[1-P(D)]$ measure increased from 0.15 when recall of only 4 items was averaged prior to computing $\mathrm{P}(\mathrm{STS})$ to 0.71 and 1.38 when recall of 8 and 16 items, respectively, were averaged. Most of this increase in estimated STS was attributable to obtaining negative scores which were larger in magnitude than the largest possible positive score when only a few recall scores were averaged prior to computing $\mathrm{P}$ (STS).

In order to avoid obtaining large negative scores in the present experiment, 24 observations ( 2 positions $x$ 2 trials $\times 6$ subjects) were averaged prior to computing $[P(I)-P(D)] /[1-P(D)]$ and $[P(I)-P(M)] /$ $[1-P(M)]$ measures. The same 24 observations were averaged after computing other measures of STS, so most measures could be compared with each other in the same analysis of variance.

The P(STS) was analyzed by a 2 by 2 by 3 by 3 by 8 analysis of variance, with arrangement (nominal or functional), data (presentation or rehearsal), presentation time $(1.25,2.50$, or $5.00 \mathrm{sec})$, position (13 to 18 with two adjacent positions averaged), and measure (recency, intervening different item, intervening same item, last rehearsed (presented) first recalled, first recall, $P(I)-P(D),[P(I)-P(D)] /$ $[1-P(D)]$, or $[P(1)-P(M)] /[1-P(M)])$ as withinsubject factors. This analysis indicated that the Measure by Position, $F(1,5)=16.35, \mathrm{p}<.01$, Presentation Time by Position, $F(2,8)=5.97$, $\mathrm{p}<.05$, Arrangement by Data by Position, $\mathrm{F}(1,5)=$ 7.78. $p<.05$, and Arrangement by Data by Presentation Time by Measure, $F(1,5)=10.16$, $\mathrm{p}<.05$, interactions were significant.

Despite changes in arrangement, data, presentation time, and measure, the P(STS) alwavs increased across terminal positions. The Measure by Position interaction resulted from the recency measure yielding a higher estimate of STS than all other measures for early (but not late) terminal positions, and from the $P(I)-P(D)$ measure yielding a smaller estimate of STS than all other measures for late (but not early) terminal positions. The Presentation Time by Position interaction was due to the longest presentation time decreasing $\mathrm{P}(\mathrm{STS})$ for the last terminal positions, but not earlier terminal positions. And the Arrangement by Data by Position interaction resulted from the difference between nominal and functional arrangements increasing across early terminal positions when $\mathrm{P}(\mathrm{STS})$ was computed with rehearsal (but not presentation) data.

The Arrangement by Data by Presentation Time by Measure interaction is illustrated in Figure 4. Instead of graphing the averaged P(STS) for items in the last six positions, the averaged $\mathrm{P}(\mathrm{STS})$ was multiplied by six to indicate the average number of items output from STS.

Inspection of Figure 4 indicates that, with a nominal arrangement, data had little or no effect on all measures, and increases in presentation time decreased all measures (except recency). Placing items in their functional rather than nominal positions did not alter the effect of presentation time on any measure (except first recall) when P(STS) was computed with presentation data. On the other hand, when items were in their functional positions, computing $\mathrm{P}(\mathrm{STS})$ with rehearsal rather than presentation data altered the effect of presentation time on all measures (except first recall, $P(I)-P(D)$, and $[P(I)-P(D)] /[1-P(D)])$. That is, unlike a nominal arrangement, with a functional arrangement increases in presentation time increased recency, intervening different item, and $[\mathbf{P}(\mathbf{I})-\mathbf{P}(\mathbf{M})] /$ 1 - P(M)] measures, and had little or no effect on intervening same-item and last-rehearsed/first- 
recalled measures when $\mathrm{P}(\mathrm{STS})$ was computed with rehearsal data.

By definition, all measures shown in Figure 4 (except first recall, $P(I)-P(D)$, and $[P(I)-P(D)] /$ [1 - P(D)]) limit P(STS) to terminal positions when $\mathrm{P}(\mathrm{STS})$ is computed with presentation or rehearsal data and items are in their nominal or functional positions, respectively. Hence, summing P(STS) across all positions rather than just terminal positions could only alter the nominal-presentation and functional-rehearsal curves shown in Figure 4 for first recall, $P(I)-P(D)$, and $[P(I)-P(D)] /[1-P(D)]$ measures.

With a first recall measure, summing P(STS) across all positions increased estimated STS output by increasing nominal-presentation and functionalrehearsal curves to a constant three items at each presentation time. Like first recall, summing P(I) -P(D) across all positions eliminated all differences between nominal-presentation and functional-rehearsal curves. On the other hand, unlike first recall, summing $P(I)-P(D)$ across all positions did not alter the estimated number of items output from STS or the effect of presentation time on STS. Finally, like $P(I)-P(D)$, summing $[P(I)-P(D)] /$ $[1-P(D)]$ across all positions did not alter the effect of presentation time on STS. However, unlike both $\mathrm{P}(\mathrm{I})-\mathrm{P}(\mathrm{D})$ and first recall, summing $[\mathrm{P}(\mathrm{I})-\mathrm{P}(\mathrm{D})] /$ $[1-P(D)]$ across all positions decreased the estimated number of items output from STS, and did not eliminate differences between nominal-presentation and functional-rehearsal curves.

While summing $\mathrm{P}(\mathrm{STS})$ across all positions (rather than just terminal positions) could alter only a few nominal-presentation and functional-rehearsal curves shown in Figure 4, it could alter all nominal-rehearsal and functional-presentation curves. That is, while most measures (by definition) restrict STS to terminal positions when $\mathrm{P}(\mathrm{STS})$ is computed with presentation or rehearsal data and items are in their nominal or functional positions, respectively, no measure necessarily restricts STS to terminal positions when P(STS) is computed with presentation or rehearsal data and items are in their functional or nominal positions, respectively.

Since arrangement of items can alter the distribution of $\mathrm{P}(\mathrm{STS})$ across positions, but not the averaged $P(S T S)$ across all positions (for all measures except $[P(I)-P(D)] /[1$ - $P(D)]$, differences between nominal and functional curves in Figure 4 indicate the degree to which items had to be both one of the last six items presented and one of the last six items rehearsed before they were output from STS. That is, the nearly identical nominal and functional presentation curves (for all measures except first recall where $\mathrm{P}(\mathrm{STS})$ is computed with order of recall rather than either presentation or rehearsal data) indicates that according to most measures the last six

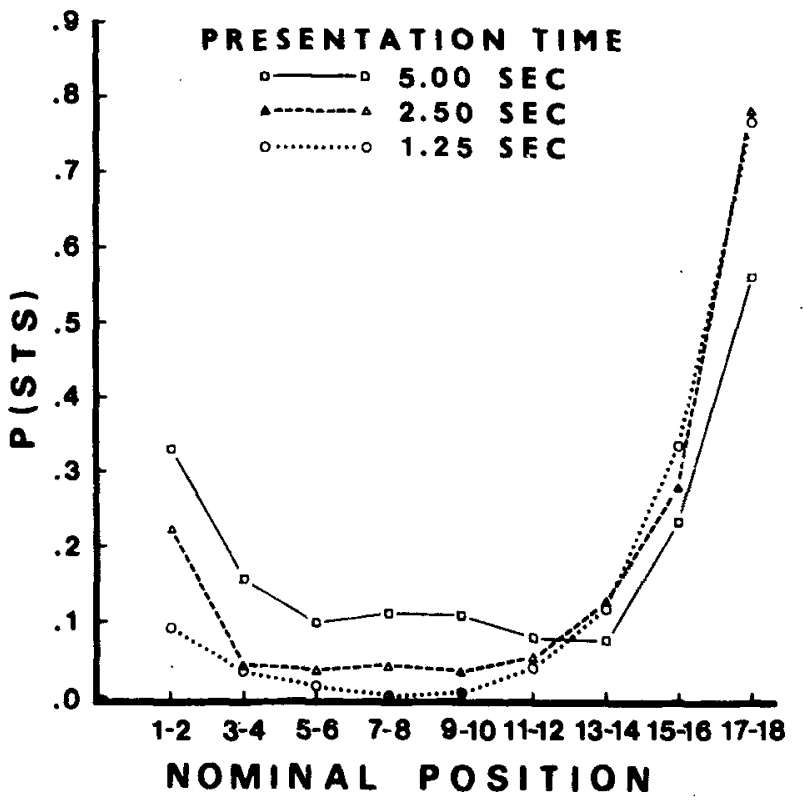

Figure 5. The $\mathrm{P}(\mathrm{STS})$ as a function of presentation time and nominal position. Except when P(STS) dropped below $0.05, \pm 1$ standard error of the mean remahed constant across presentation times and positions at \pm 0.06 .

items presented were output from STS only if they were one of the last six items rehearsed. Conversely, the large differences between nominal and functional rehearsal curves (for all measures except $P(I)-P(D)$ and $[P(I)-P(D)] /[1-P(D)])$ indicates that according to most measures the last six items rehearsed were output from STS even if they were not one of the last six items presented. Moreover, inspection of the rehearsal curves in Figure 4 indicates that increases in presentation time increased the probability that items presented prior to the last six were output from STS, and decreased the probability that the last six items presented were output from STS. This shift in P(STS) across nominal positions as a function of presentation time is also illustrated in Figure 5. Moreover, unlike Figure 4, Figure 5 illustrates the distribution of P(STS) across preterminal nominal positions. Inspection of Figure 5 indicates that when P(STS) was computed with rehearsal data, the P(STS) decreased across the first few nominal positions, remained constant across middle positions, and increased across terminal positions. The P(STS) in Figure 5 was averaged across intervening different-item, intervening same-item, last-rehearsed/first-recalled, firstrecall, recency, and $[P(I)-P(M)] /[1-P(M)]$ measures, because each of these measures yielded the same pattern of results. On the other hand, P(STS) was not averaged across $P(I)-P(D)$ and $[P(I)-P(D)] /$ $[1-P(D)]$ measures because they indicated that no items in preterminal nominal positions were output from STS.

The only measures which were not included in the above analysis of STS are intercept and intersection. 
Unlike all the above measures, it is necessary to vary list length to determine the effect of presentation time on these measures. Since list length was not varied, only the effects of arrangement (nominal or functional) and item (all or terminal) can be determined.

Like intervening item, last-rehearsed/first-recalled, recency, and $[P(I)-P(M)] /[1-P(M)]$ measures, the intercept measure indicated that about three terminal items were output from STS. Unlike all measures except first recall, however, summing data across all items (rather than just terminal items) increased estimated STS output by increasing the intercept from 3.06 to 5.28 . Moreover, unlike all measures except $\mathrm{P}(\mathrm{I})$ - $\mathrm{P}(\mathrm{D})$, the intercept did not vary as a function of arrangement.

An intersection measure yields either a higher, lower, or same estimate of STS as an intercept measure, depending on whether the slope is positive, negative, or zero, respectively. The slope is conventionally computed from the effect on recall of changes in total presentation time with changes in list length. However, since list length was not varied in the present experiment, the slope was determined from the effect on recall of changes in total presentation time with changes in presentation rate.

The slope was practically zero for the last six items presented $(-0.008)$, but positive for all items $(0.029)$ as well as for the last six items rehearsed $(0.054)$. The zero slope suggests that, like an intercept measure, with an intersection measure three of the last six items presented were output from STS. Furthermore, the equal intercepts and unequal slopes for the last six items presented and the last six items rehearsed suggest that, like all measures except intercept and $P(I)-P(D)$, with an intersection measure an item was more likely to be output from STS if it was one of the last six items rehearsed than if it was one of the last six items presented. Finally, comparison of the slope and intercept for all items with the slope and intercepts for terminal items suggests that, like intercept and first recall measures, with an intersection measure summing data across all items (rather than just terminal items) increases STS output.

\section{DISCUSSION}

\section{Measures of STS}

As is not surprising, the results indicate that all measures of STS do not yield identical results. The best measures of STS can be determined by comparing the effects of nominal position, functional position, and presentation time on measures of STS with the effect of these variables on theoretical STS.

First, consider the effect of nominal position on theoretical STS. Memory dichotomy theorists have assumed that rehearsal generates a STS trace, and items in STS are one of the first items recalled. As is evident from the effect of nominal position on intervening item and last-rehearsed/first-recalled measures (see Figure 5), some of the first items presented were both one of the last items rehearsed and one of the first items recalled immediately following list presentation. Hence, all measures of STS should indicate that some of the first items presented were output from STS. When P(STS) was computed with presentation data, however, all measures (except first recall, intercept, and intersection) failed to exhibit these results. On the other hand, when P(STS) was computed with rehearsal data, only $P(I)-(P D)$ and $[P(I)-P(D)] /$ $[1-P(D)]$ measures failed to exhibit the predicted results.

Second, consider the effect of functional position on theoretical STS. Memory dichotomy theorists have assumed that $\mathrm{P}(\mathrm{STS})$ decays rapidly after last rehearsal of an item. Thus, measures of STS should indicate that the P(STS) is zero for items which received their last rehearsal early during list presentation. Only 5 of the 10 measures examined did not limit by definition $\mathrm{P}(\mathrm{STS})$ to the last items rehearsed when $\mathrm{P}$ (STS) was computed with rehearsal data. Two of these measures-P(I) - P(D) and $[P(I)-P(D)] /[1-P(D)]$-indicated that no items which had received their last rehearsal early during list presentation were output from STS. On the other hand, the remaining three measures-first recall, intercept, and intersection-failed to restrict STS to the last items rehearsed.

Finally, consider the effect of presentation time on theoretical STS. Memory dichotomy theorists have assumed that $\mathrm{P}(\mathrm{STS})$ at list termination varies as a function of either time or number of different items intervening between last rehearsal of an item and list termination. Consequently, the effect of presentation time on STS can be derived from inspection of time and different-item data in Figures 2 and 3. Inspection of these figures indicates that increases in presentation time increased time and number of different items intervening between last rehearsal of the last six items presented and list termination. Hence, increases in presentation time should decrease $\mathrm{P}(\mathrm{STS})$ for these items. Inspection of Figure 4 indicates that all measures except recency exhibited this decline.

Inspection of Figure 2 also indicates that when items were in their functional positions, increases in presentation time only slightly increased time between last rehearsal of an item and list termination. Moreover, when items were in their functional positions, increases in presentation time did not alter the number of items rehearsed after each item. Hence, increases in presentation time should produce smaller reductions in $\mathrm{P}(\mathrm{STS})$ for the last six items rehearsed than for the last six items presented. Inspection of Figure 4 indicates that most measures failed to exhibit the predicted results. For example, contrary to predictions, with $P(I)-P(D)$ and 
$[P(I)-P(D)] /[1-P(D)]$ measures, increases in presentation time produced the same reduction in STS for the last six items rehearsed as that observed for the last six items presented. Furthermore, while recency, $[\mathrm{P}(\mathrm{I})-\mathrm{P}(\mathrm{M})] /[1-\mathrm{P}(\mathrm{M})]$, and intervening differentitem measures indicated that presentation time affected P(STS) for the last six items rehearsed differently than P(STS) for the last six items presented. contrary to predictions they indicated that increases in presentation time yielded large increases in STS for the last six items rehearsed.

The above results indicate that (a) intervening sameitem and last-rehearsed/first-recalled measures came closer than any other measures examined to exhibiting the predicted effects of nominal position, functional position, and presentation time on theoretical STS, and (b) computing P(STS) with presentation data yields a biased estimate of STS. Hence. investigators who want to estimate P(STS) should compute $\mathrm{P}(\mathrm{STS})$ with rehearsal data, using either an intervening same-item measure or a last-rehearsed/first-recalled measure.

\section{Recall}

The recall results replicate the following four findings. First. immediate free recall is a $\mathrm{U}$-shaped function of an item's nominal position (Glanzer \& Cunitz. 1966: Murdock, 1962). Second, a small increase in the list retention interval decreases recall of itens in terminal. but not preterminal. nominal positions (Glanzer \& Cunitz, 1966; Glanzer et al., 1969; Postman \& Phillips, 1965). Third, increases in presentation time increase immediate free recall of items in preterminal, but not terminal, nominal positions (Craik \& Levy, 1970; Glanzer \& Cunitz. 1966: Murdock, 1962). And fourth, increases in presentation time increase delayed recall of items in each nominal position (Raymond, 1969).

In addition to replicating the above four recall findings, the results extend each of these findings by indicating whether arranging items in their functional positions alters the effect of variables on the free recall curve. Specifically. like a nominal arrangement, with a functional arrangement a small increase in the list retention interval decreased recall of items in terminal. but not preterminal, positions. Moreover, while increases in presentation time did not increase delayed recall of items in beginning functional positions. like a nominal arrangement, with a functional arrangement increases in presentation time increased delayed recall of items in middle and terminal positions. On the other hand. unlike a nominal arrangement, with a functional arrangement increases in presentation time increased immediate free recall of items in terminal, but not beginning, positions. Finally, arranging items in their functional rather than nominal positions eliminated reductions in recall across beginning positions, and augmented increases in recall across terminal positions.

The finding that position and presentation time have different effects on nominal free recall curves than on functional free recall curves has been replicated by the author in four different experiments. The effects of position and presentation time on nominal free recall curves can be explained from the effects of position and presentation time on time subjects spend trying to learn each item and on the length of each item's retention interval (Brodie, 1973). The same explanation can account for most of the effects of position and presentation time on functional recall curves. The multiple correlation between the mean recall scores shown in Figure 1 and both mean study time and mean item retention interval was 0.901 where recall equals 0.590 plus 0.224 times the logarithmic value of study time in seconds minus 0.151 times the logarithmic value of an item's retention interval in seconds.

Since some manipulations affect functional free recall curves differently than nominal recall curves, and since items in STS are more likely to be in terminal positions with a functional rather than nominal arrangement, the recall data support the conclusion that items must be placed in their functional positions before deciding whether the effect of a given manipulation on recall either supports or impugns the existence of separate STS and LTS. Hence, the present data on recall and measures of STS clearly support Wickelgren's (1973) contention that it is necessary to take into account subjects' rehearsal and their order of recall before trying to infer the existence of separate stores from free recall data.

\section{REFERENCES}

Anderson, C. M. B., \& Craik, F. I. M. The effect of concurrent task on recall from primary memory. Journal of Verbal Leaming and Verbal Behavior, 1974, 13, 107-113.

Atkinson, R. C.. \& Shiffrin. R. M. Human memoty: A proposed system and its control processes. In $K$. W. Spence and J. T. Spence (Eds.). The psychology of learning and motivation: Advances in research and theory (Vol. 2). New York: Academic Press, 1968. Pp. 89-195.

Atkinson, R. C., \& Shiffrin, R. M. The control of short-term memory. Scientific American, 1971, 225, 82-90.

BADDELEY, A. D. Estimating the short-term component in free recall. British Journal of Psychology, 1970, 61. 13-15.

Baddeley, A. D., Scott, D., Drynan, R., \& Smrth, J. C. Shortterm memory and the limited capacity hypothesis. British Journal of Psychology, 1969, 60, 51-55.

Brodie. D. A. Are effects of serial position and presentation time on free recall mediated by changes in rehearsal? Doctoral dissertation. University of Western Ontario, London. 1973.

Corballis, M. C. Patterns of rehearsal in immediate memory. British Journal of Psychology, 1969, 60, 41-49.

Craik, F. I. M. Two components in free recall. Journal of Verbal Learning and Verbal Behavior, 1968, 7, 996-1004. 
Craik, F. I. M. Primary memory. British Medical Bulletin 1971, 27, 232-236.

Craik, F. I. M., \& Birtwistle, J. Proactive inhibition in free recall. Journal of Experimental Psychology, 1971, 91, 120-123.

CraIK, F. I. M., \& LeVY, B. A. Semantic and acoustic information in primary memory. Journal of Experimental Psychology. 1970, 86, 77-82.

Glanzer, M. Storage mechanisms in recall. In G. H. Bower (Ed.). The psychology of learning and motivation: Advances in research and theory (Vol. 5). New York: Academic Press, 1972. Pp. 129-193.

Glanzer, M., \& Cunitz, A. R. Two storage mechanisms in free recall. Journal of Verbal Learning and Verbal Behavior, 1966, 5, 351-360.

Glanzer. M., Gianutsos, R., \& Dubin, S. The removal of items from short-term storage. Journal of Verbal Learning and Verbal Behavior, 1969, 8, 435-447.

GlanZER, M., \& SchWARTz, A. Mnemonic structure in free recall: Differential effects on STS and LTS. Journal of Verbal Learning and Verbal Behavior, 1971, 10, 194.198.

Kučera, H., \& Francis, W. N. Computational analysis of present-day American English. Providence, R.I: Brown Univerity Press, 1967

Levy, B. A., \& Baddeley, A. D. Recall of semantic clusters in primary memory. Quarterly Joumal of Experimental Psychology, 1971, 23, 8-13.

MuRDOCK, B. B., JR. The immediate retention of unrelated words. Journal of Experimental Psychology, 1960, 60, 222-234.

Murdock, B. B., JR. The serial position effect in free recall. Journal of Experimental Psychology, 1962, 64, 482-488.

MURDock, B. B., JR. Recent developments in short-term memory. British Journal of Psychology, 1967, 58, 421-433.

MYERS, J. L. Fundamentals of experimental design (2nd ed.). Boston: Allyn and Bacon, 1972.
Postman, L., \& Phillips, L. W. Short-term temporal changes in free recall. Quarterly Journal of Experimental Psychology, 1965, 17, 132-138.

RAYMond, B. Short-term storage and long-term storage in free recall. Journal of Verbal Learning and Verbal Behavior, 1969, 8. $567-574$.

Reitman, J. S. Without surreptitious rehearsal, information in short-term memory decays. Journal of Verbal Learning and Verbal Behavior. 1974, 13. 365-377.

Rundus, D., \& Atrinson, R. C. Rehearsal processes in free recall: A procedure for direct observation. Joumal of Verbal Learning and Verbal Behavior, 1970, 9, 99-105.

Silverstein. C., \& Glanzer, M. Concurrent task in free recall: Differential effects of LTS and STS. Psychonomic Science, 1971, 22, 367-368.

THORNDIKE, E. L., \& LORGE, I. The teacher's word book of 30.000 words. New York: Columbia University Press, 1944

Tulving, E., \& Colotra, V. A. Free recall of trilingual lists. Cognitive Psychology, 1970, 1, 86-98.

Tulving, E., \& Patterson, R. D. Functional units and retrieval processes in free recall. Journal of Experimental Psychology. 1968, 77, 239-248.

WATKINs, M. J: Concept and measurement of primary memory. Psychological Bulletin, 1974, 81, 695-711.

Waugh, N. C., \& Norman, D. A. Primary memory. Psychological Review, 1965, 72, 89.104.

Wickelgren, W. A. The long and the short of memory. Psychological Bulletin, 1973, 80, 425-438.

(Received for publication November 15, 1974; revision received March 3, 1975.) 